\title{
Accessibility Research of Highway Network System in Henan Province
}

\author{
Kai Guang ZHANG ${ }^{1, a}$, Ming Ting BA ${ }^{1, b}$ and Hong Ling MENG ${ }^{1, c}$ \\ ${ }^{1}$ Institute of 3S Technology, Zhengzhou Normal University, Zhengzhou 450044, China
}

\begin{abstract}
The accessibility index is designed by using the inverse of time distance and population weighted average value to research the city accessibilities in a region, with the definition of time threshold, which not only considers the transportation characteristics of each segment in the region, but also effectively reduces the digital effects from measurement on the analysis results, makes the analysis result could better reflect the reality at the same time. The results show that the Henan highway traffic network is relatively perfect, basically have been forming a high speed transportation system with the provincial capital, Zhengzhou, as the center. The city accessibility indexes in Henan province show the characteristics of normal distribution, the Accessibility differences are relatively obvious, the regional distribution of highway is very unbalanced. The accessibilities have high-high and low-low aggregation phenomenon, the Accessibility along the north to South and northwest to southeast direction becomes worse; the accessibility distribution and population distribution exist obvious departure.
\end{abstract}

\section{Introdution}

The accessibility means the interactions between the nodes in a traffic network and the traffic convenience from one node to others, which is an Important tools to evaluate the maturity of a regional traffic network structure [1-3], is firstly proposed by Dr. Hansen to study its impact on landuse in a region. In 1990, Professor Murayama studied the impact of the railway development on city accessibilities in Japan, and got some meaningful results[4]. Since the 1980s, Fractal theory, network theory, space syntax, shortest path, spatial topology were introduce in the study of city accessibility, which not only enriched the connotation of accessibility study, but also got some valuable research results. However these studies mainly focused on simple traffic network and used Euclidean distance measurement [5-14].With a complex highway traffic network gradually forming in China, city links gradually are becoming more diversity, national highway, fast-track lines, and more than one highway lines with different traffic characteristics constitute the main link between the cities in a region. The efficient highway traffic network closes the distance between cities, as well as gives a new connotation on the city accessibility research [15-18].

Henan province is the main body of Central Plains Economic Region and an important traffic hub in China. By the end of 2015, highway mileage is more than 6600 $\mathrm{km}$ forming a relatively well-developed highway transportation system with 9 national highway lines as the backbone, 22 local highway lines to complement. The developed highway has changed a lot in people's life style and greatly boosted the local economy development [19].

In the paper, based on Henan Province Ordinary traffic network, a new accessibility index is constructed by using the inverse of time distance and populationweighted average value, to evaluate the accessibilities between cities in the province and the sophistication of highway traffic network, in order to provide scientific reference for the region to further improve the network. Spatial data in the research comes from Henan traffic network database [19], the time measure bases on the empirical data of travelling through road junctions and segments between January to September in 2015, the shortest time distance between two points is calculated by NEDS algorithm[20].

\section{City accessibility index}

\section{1 accessibility index}

The city accessibility index in a highway traffic network is a degree of accessibility from one city to all adjacent cities, usually by using the weighted average distances deriving from population, regional GDP, passenger and freight traffic transport amount as the weighted, the optimum route number, the sum of shortest distance, they describe the numerical characteristics of city accessibility in different aspects[5-15]. However, for the complex highway traffic network as in China recently, the national highway traffic network is being intensified by the provinces (municipalities), two adjacent cities have

\footnotetext{
a Corresponding author: zzgis@126.com
} 
multiple highway lines and fast track lines to link, these descriptions hide some limitations.

Different highway line has different transportation characteristics with features complement each other, not a substitute for the function. Therefore, only using numerical characteristics of single line or the number of general lines to describe the correlation between cities is not comprehensive solution to analyze the whole city accessibility in a region. Highway traffic characteristics is an important attribute of highway traffic, mainly reflect on the comfortableness of driving and the average speed of traffic, the last could be is expressed as the time distance between the two points in time measurement.

There are various possibilities in direct connections and circuitous connections between two cities. In the study of city accessibility coming from highway traffic network, it is necessary to set a time threshold by only considering traffic routes within the threshold range. In the paper, the time threshold is set as the shortest travel time between cities on the ordinary traffic network(Correspond to highway traffic network), two routes on the highway traffic network are considered to be the same if their repetition rate is greater than $50 \%$.

In this paper, the weighted inverse of time distance is used to carry on the city accessibility research. The accessibility from city $i$ to city $j$ is defined as the sum of the time inverse distance for all routes which fall in the time threshold range, and do not travel through the third city, denoted as $\boldsymbol{A}_{i j}$.

$$
A_{i j}=\sum \frac{1}{T_{r}}
$$

The larger $\boldsymbol{A}_{i j}$ means better accessibility form $i$ to $\boldsymbol{j}$. Here we should be paid more attention on $\boldsymbol{A}_{i j} \neq \boldsymbol{A}_{j i}$.

The city accessibility $\boldsymbol{A}_{i}$ within a region is defined as the weighted average of accessibility from all the other cities in the region to it,

$$
A_{i}=\sum_{r=1}^{n} \frac{M_{j} A_{j i}}{M_{j}},
$$

where, $\boldsymbol{M}_{\boldsymbol{j}}$ is the weight of the city $\boldsymbol{j}$ indicating the influence degree of $\boldsymbol{j}$ on the accessibility of other cities in the region. Generally $\boldsymbol{M}_{j}$ used the residents population. The larger $\boldsymbol{A}_{\boldsymbol{i}}$ means the better the accessibility of $\boldsymbol{i}$ is, and there are more attractiveness for the adjacent cities.

\subsection{Probability distribution of accessibility}

The probability distribution of accessibility describes the spatial distribution characteristics of accessibility in a region for all cities. The mean $\boldsymbol{E}$ indicates the average traffic capacity, the standard deviation $\sigma$ and the range $\boldsymbol{Z}$ indicate the difference degree of traffic capacity in a region. The larger $\sigma$ and $\boldsymbol{Z}$ are, the more significant the regional capacity difference is, the more departure between the distribution of highway traffic network and the distribution of population is in space.

$$
E=\frac{1}{n} \sum_{r=1}^{n} A_{r}, \quad D=\frac{1}{n-1} \sum_{r=1}^{n}\left(A_{r}-E\right)^{2}, \quad Z=\max \left(A_{i}\right)-\min \left(A_{i}\right)
$$

\subsection{Centrality correlation analysis of accessibility}

The city accessibilities coming from highway traffic network in a region have some correlations with the location of cities and the weighted parameters. The accessibility of one city reflects its centrality degree in the region, and the convenience degree the people of adjacent cities traveling to it. The center of accessibility $\boldsymbol{B}_{0}$ in a region is defined as the weighted average of city geographical coordinates, the center of population $\boldsymbol{R}_{0}$ is defined as the weighted average of the number of residents population with its Accessibility as weight.

$$
\begin{aligned}
& B_{0}=\left(\frac{A_{i}}{\sum A_{i}} x_{i}, \frac{A_{i}}{\sum A_{i}} y_{i}\right) \\
& R_{0}=\left(\frac{m_{i}}{\sum M_{i}} x_{i}, \frac{m_{i}}{\sum M_{i}} y_{i}\right)
\end{aligned}
$$

where $\left(\boldsymbol{x}_{\boldsymbol{i}}, \boldsymbol{y}_{\boldsymbol{i}}\right)$ represents the geographical coordinates of the city $i$.

\section{City accessibility analysis in Henan province}

\subsection{Time threshold in Henan province}

The time threshold in the analysis of accessibility coming from highway traffic network is defined as the minimum time distance between two cities traveling in ordinary traffic network consisting of national ordinary trunk way (State Road), provincial ordinary trunk way (provincial road) (see Figure 1.), the calculation results are shown in Table1 (unit: minutes).

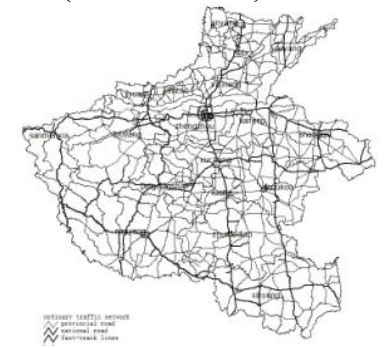

Figure 1 Ordinary traffic network

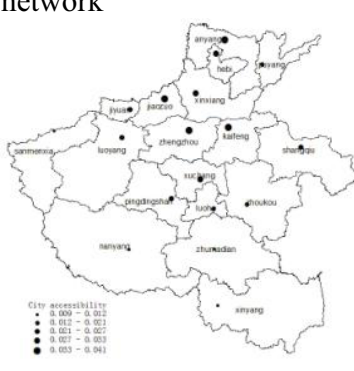

Figure 3. The distribution of city accessibility

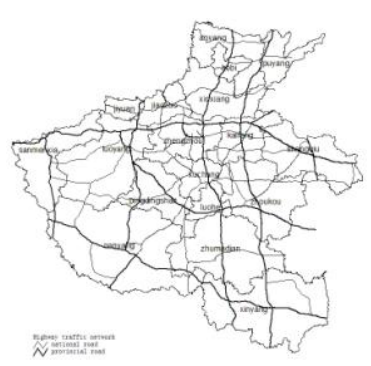

Figure 2 Highway traffic network

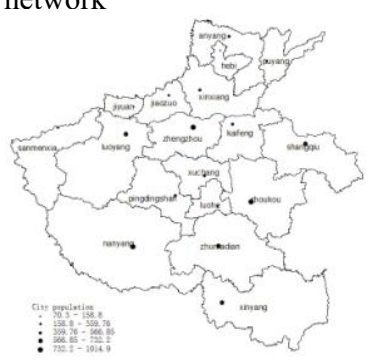

Figure 4. The distribution of population

\subsection{City accessibility in Henan province}

The calculation of accessibility indexes from city $j$ to $i$ is based on highway traffic network (Figure 2.) by using 
formula(1), then calculate the shortest routes from city $j$ to $i$ and their traveling times which range in the time threshold, the results are shown in Table 2, then using formula (2), the number of residents population of $j$ as the weight calculate the accessibility of city $i$, the results are shown in Table 3.

\subsection{The spatial distribution of city accessibilities in Henan province}

As shown in Figure 3., there is a local aggregation features in the spatial distribution of city accessibilities, presenting high-high aggregation in the central and the northern area, as well as low-low aggregation in the southern and the eastern area in the province. From the north to south and the northwest to southeast, it gradually deteriorated. Although the residents population weights are used to calculate the accessibility indexes, there is a significant deviation between the spatial distribution of accessibility and the spatial distribution of residents population (Figure 4.), the most significant region is in the southern and the central area in the province.

Table1. The time threshold in Henan province

\begin{tabular}{|c|c|c|c|c|c|c|c|c|c|c|c|c|c|c|c|c|c|c|}
\hline & ZZ & KF & LY & PDS & AY & HB & XX & JZ & PY & XC & LH & SMX & NY & SQ & XY & ZK & ZMD & JY \\
\hline ZZ & & 59 & 150 & 203 & & & 84 & 134 & & 88 & & & & & & 228 & & 166 \\
\hline KF & 59 & & & & & & 106 & & 232 & & & & & 140 & & 220 & & \\
\hline LY & 150 & & & 168 & & & & 134 & & 221 & 283 & 175 & 329 & & & & & 91 \\
\hline PDS & 203 & & 168 & & & & & & & 121 & 163 & & 199 & & 318 & & & \\
\hline AY & & & & & & 48 & & & 150 & & & & & & & & & \\
\hline HB & & & & & 48 & & 71 & & 166 & & & & & & & & & \\
\hline XX & 84 & 106 & & & & 71 & & 128 & 199 & & & & & & & & & \\
\hline JZ & 134 & & 134 & & & & 128 & & & & & & & & & & & 76 \\
\hline PY & & 232 & & & 150 & 166 & 199 & & & & & & & & & & & \\
\hline XC & 88 & & 221 & 121 & & & & & & & 66 & & & 237 & 226 & 133 & & \\
\hline LH & & & 283 & 163 & & & & & & 66 & & & 256 & & & 102 & 70 & \\
\hline SM & & 175 & & & & & & & & & & 375 & & & & & \\
\hline NY & & & 329 & 199 & & & & & & & 256 & 375 & & & 215 & & 225 & \\
\hline SQ & & 140 & & & & & & & & 237 & & & & & & 214 & & \\
\hline XY & & & & 318 & & & & & & 226 & & & 215 & & & 255 & 103 & \\
\hline ZK & 228 & 220 & & & & & & & & 133 & 102 & & & 214 & 255 & & 156 & \\
\hline ZM & & & & & & & & & & & 70 & & 225 & & 103 & 156 & & \\
\hline JY & 166 & & 91 & & & & & 76 & & & & & & & & & & \\
\hline
\end{tabular}

Table 2. The accessibilities between cities in Henan province

\begin{tabular}{|l|l|l|l|l|l|l|l|l|l|l|l|l|l|l|l|l|l|l|}
\hline & ZZ & KF & LY & PDS & AY & HB & XX & JZ & PY & XC & LH & SMX & NY & SQ & XY & ZK & ZMD & JY \\
\hline ZZ & & 0.058 & 0.049 & 0.022 & & & 0.037 & 0.056 & & 0.049 & & & & & & 0.020 & & 0.015 \\
\hline KF & 0.058 & & & & & & 0.030 & & 0.014 & & & & & 0.038 & & 0.022 & & \\
\hline LY & 0.049 & & & 0.034 & & & & 0.029 & & 0.018 & 0.008 & 0.020 & 0.004 & & & & & 0.042 \\
\hline PDS & 0.022 & & 0.034 & & & & & & & 0.035 & 0.017 & & 0.023 & & 0.007 & & & \\
\hline AY & & & & & & 0.040 & & & 0.034 & & & & & & & & & \\
\hline HB & & & & & 0.040 & & 0.031 & & 0.024 & & & & & & & & & \\
\hline XX & 0.037 & 0.030 & & & & 0.031 & & 0.033 & 0.013 & & & & & & & & & \\
\hline JZ & 0.056 & & 0.029 & & & & 0.033 & & & & & & & & & & & 0.030 \\
\hline PY & & 0.014 & & & 0.034 & 0.024 & 0.013 & & & & & & & & & & & \\
\hline XC & 0.049 & & 0.018 & 0.035 & & & & & & & 0.027 & & & 0.018 & 0.007 & 0.047 & & \\
\hline LH & & & 0.008 & 0.017 & & & & & & 0.027 & & & 0.010 & & & 0.030 & 0.022 & \\
\hline SM & & & 0.020 & & & & & & & & & & 0.005 & & & & & \\
\hline NY & & & 0.004 & 0.023 & & & & & & & 0.010 & 0.005 & & & 0.009 & & 0.008 & \\
\hline SQ & & 0.038 & & & & & & & & 0.018 & & & & & & 0.023 & & \\
\hline XY & & & & 0.007 & & & & & & 0.007 & & & 0.009 & & & 0.006 & 0.017 \\
\hline ZK & 0.020 & 0.022 & & & & & & & & 0.047 & 0.030 & & & 0.023 & 0.006 & & 0.010 & \\
\hline ZM & & & & & & & & & & & 0.022 & & 0.008 & & 0.017 & 0.010 & & \\
\hline JY & 0.015 & & 0.042 & & & & & 0.030 & & & & & & & & & & \\
\hline
\end{tabular}

Table 3. The accessibilities in Henna province

\begin{tabular}{|l|l|l|l|l|l|l|l|l|l|l|l|l|l|l|l|l|l|}
\hline ZZ & KF & LY & PDS & AY & HB & XX & JZ & PY & XC & LH & SMX & NY & SQ & XY & ZK & ZMD & JY \\
\hline
\end{tabular} \begin{tabular}{|l|l|l|l|l|l|l|l|l|l|l|l|l|l|l|l|l|l|}
\hline 0.038 & 0.035 & 0.025 & 0.023 & 0.036 & 0.033 & 0.031 & 0.041 & 0.021 & 0.030 & 0.018 & 0.011 & 0.010 & 0.026 & 0.009 & 0.020 & 0.012 & 0.027 \\
\hline
\end{tabular}

\subsection{Numerical characteristics of city accessibilities in Henan province}

There are nine cities whose city accessibility are more than the mean $\boldsymbol{E}=\mathbf{0 . 0 2 4 9}$ and nine cities whose city accessibility are less than it. Jiaozuo is the most optimal accessibility city and Xinyang is the poorest accessibility city in the region, all of them are located in the border area of the province. The standard deviation and range respectively are $\sigma=\mathbf{0 . 0 1 0 4}$ and $\boldsymbol{Z}=\mathbf{0 . 0 3 7 1}$ showing that 
the difference of city accessibility is relatively obvious, and the spatial distribution of highway is relatively unbalanced in the province.In Figure 5. the solid lines are the fitted curve of probability distribution for the standardized city accessibilities, and the dash curves are standardized normal distribution. The figure shows that the city accessibility indexes and the normal distribution fit very well, By hypothesis testing $\chi^{2}$ and under the confidence of $\mathbf{0 . 1 0}$, the probability distribution of city accessibility indexes meets the normal distribution.

\subsection{Centrality correlation analysis of city accessibilities in Henan province}

Accessibility center and the population distribution center of Henan province respectively are located in the northern part of Zhengzhou city and the northern part of Xuchang City ( Figure 6., Figure 7.), there is $52 \mathrm{~km}$ between them. If the range of the accessibility and population are divided into 5 levels from large to small, and use the linear interpolation

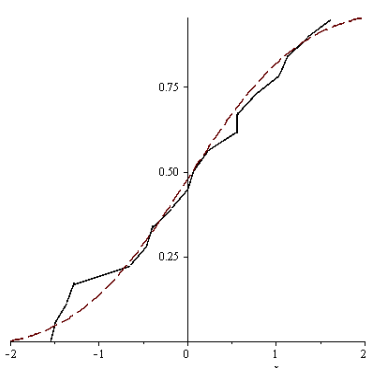

Figure 5. The probability distribution of urban accessibility method,we can get the spatial patterns of them as Figure 6., Figure 7.

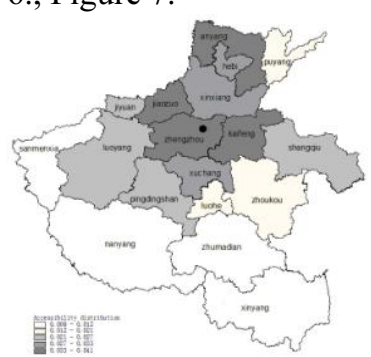

Figure 6. The spatial pattern of urban accessibility

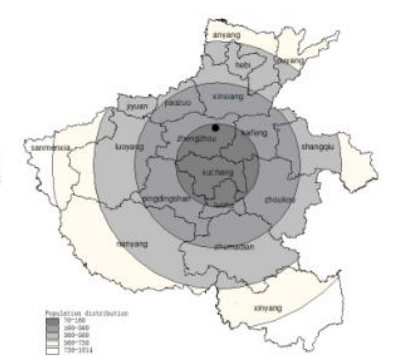

Figure 7. The spatial pattern of urban population
Contrasting Figure 6., Figure 7., It could be found that the central area of the population spatial distribution pattern fits with the central region of the accessibility spatial pattern in Zhengzhou and Kaifeng very well, but in other regions there are big differences. Unlike the population spatial pattern, the accessibility spatial pattern shows irregular distribution characteristics, the reason is that the city accessibility is not only affected by the condition of the highway traffic network, but also affected by the geographical position of the city.

\section{Conclusion}

(1) By using the inverse of time distance traveling through road junctions and segments and population weighted average value to research the city accessibilities in a region, with the definition of time threshold, which not only considers the characteristics of highway traffic network, but also effectively reduces the digital effects from measurement on the analysis results, makes the analysis result could better reflect the reality at the same time.

(2) There is a relatively perfect regional highway traffic network in Henan province basically forms a high speed transportation system with the capital, Zhengzhou, as the center. Compared with other domestic provinces (municipalities), the city accessibility is better.

(3) The city accessibility indexes in Henan province show the characteristics of normal distribution $\boldsymbol{N}(\mathbf{0 . 0 2 4 9 , 0 . 0 1 0 4 )}$, the Accessibility differences are relatively obvious, the regional distribution of highway is very unbalanced.

(4) The accessibilities have high-high and low-low aggregation phenomenon, the Accessibility along the north to South and northwest to southeast direction becomes worse;

(5)The accessibility spatial distribution and population spatial distribution exist obvious departure. the most obvious areas are in the south central part of Henan province.

\section{References}

1. W. G. Hansen, Journal of the American institute of planners, 25, 73(1959).

2. L. Pinghua, L. Yuqi, Progress in geography, 24 (3), 69(2005).

3. R. Henrike, V. Amaya, Growth and Change, 43(4), 667(2012).

4. L. Qinghua, W. Xinyan, Journal of Tibet University (Natural Science), 28 (1), 117(2013).

5. S. Jinghong, L. Yuqi, L. Xiaoji, Geographical Research, 31 (7), 1280(2012).

6. X. Jingge, Z. Tinggang, Journal of Geo-Information Science, 17 (1), 54(2015).

7. L. Baojie, G. Hehe, J. Yazhou, Human Geography, 6, 76(2012).

8. X. Xu, C. Xiaoshu, Geographical Research, 26 (1): 180(2007).

9. L. Yating, Q. Yaoche, Y. Weiyang, Areal Research and Development, 29 (1),60(2010).

10. L. Hong, L. Xiaoyan, W. Chunguo, Areal Research and Development, 30 (1), 55(2011).

11. J. Awei, Progress in Geography, 31(12), 1591(2012).

12. C. Xiaoshu, X. Desheng, Y. XiaoPei, Acta Geographica Sinica, 60 (6), 903(2005).

13. J. Fengjun, W. Jiaoe, Acta Geographica Sinica, 59 (2), 29(2004).

14. W. We, C. Youhui, Acta Geographica Sinica, 61 (10), 1065(2006).

15. Y. Pei, highway, 10: 141(2013).

16. Z. Li, L. Yuqi, Acta Geographica Sinica, 61 (12), 1235(2006).

17. Z. Bing, J. Fengjun , Economic geography, 26 (5), 776(2006).

18. Z. Kaiguang, S. Yu, B. Mingting, Areal Research and Development, 33 (3), 69(2014).

19. Henan Department of Transportation. Figure and row of Henan [EB / OL] (2015-10-10) [2015-10-10]. http: //road.hncd Gov.cn/page/txhn/txhn .jsp 
20. Z. Kaiguang, M. Hongling, B. Mingting, Journal of Geomatics Science and Technology, 30 (1), 83(2013). 